\title{
Topical Gel Based Nanoparticles for the Controlled Release of Oleanolic Acid: Design and In Vivo Characterization of a Cubic Liquid Crystalline Anti- Inflammatory Drug
}

zhiqi shi ( $\nabla$ shi_mars@foxmail.com )

Changzhou Institute of Technology https://orcid.org/0000-0002-4369-1943

Shugang Pan

Changzhou Institute of Technology

Luolin Wang

Guangdong Provincial Academy of Traditional Chinese Medicine

Sha Li

wuxi hospital of traditional chinese medicine

Research

Keywords: LCNP-based gel, Oleanolic acid, Permeation studies

Posted Date: April 27th, 2021

DOl: https://doi.org/10.21203/rs.3.rs-435464/v1

License: (1) (1) This work is licensed under a Creative Commons Attribution 4.0 International License.

Read Full License 


\section{Abstract}

Background: Oleanolic acid (OA) has multiple pharmaceutical applications, but low permeability of the molecule limits its widespread use.

Methods $₫$ A cubic liquid crystalline nanoparticle (LCNP)-based gel was prepared as a potential topical delivery system for OA. The LCNP-based gel was optimized using rheological, drug release kinetic, and ex vivo permeation studies.

Results: The studies showed that the OA was trapped in the interior of the LCNP with a crystal form of Pn3m space. The optimized LCNP formulation performed well using in vitro release studies for up to $12 \mathrm{~h}$ $(85.49 \pm 0.21 \%)$. Ex vivo permeation studies showed that the LCNP-based gel formulation was superior to a standard gel formulation. The $r^{2}$ value from the Peppas equation indicated good linearity, but showed irregular (non-Fickian) diffusion, suggesting that drug release was controlled by multiple processes.

Conclusions: In this study, OA-loaded LCNPs were prepared by the precursor method, resulting in a wellcharacterized OA-LCNP gel preparation. The gel was shown to be effective in a rodent carrageenaninduced hind paw inflammation model with sustained efficacy after a single application.

\section{Introduction}

The diverse and extensive pharmacological activity of plant-based molecules has prompted scientists to consider using natural products with proven therapeutic effects as potential therapies. Many triterpenoids including $O A$ have been shown to have a wide range of pharmacological activities ${ }^{[1]}$ including being an antioxidant, hypoglycemic, anti-inflammatory, antibacterial, and hepatoprotective $e^{[2-8]}$. However, OA is a class IV drug according to the Biopharmaceutics Classification System, and has an absolute oral bioavailability of only $0.7 \%$ because of its low permeability (Papp $=1.1-1.3 \times 10^{-6} \mathrm{~cm} / \mathrm{s}$ in the apical to basolateral direction at 10 and $20 \mu \mathrm{M})$ and low aqueous solubility $(<1 \mu \mathrm{g} / \mathrm{mL})^{[9-10]}$. Several approaches including recrystallization ${ }^{[11]}$, solid dispersion ${ }^{[12]}$, nano-emulsions ${ }^{[13]}$, and drug-phospholipid complex techniques ${ }^{[14]}$ have been used to attempt to improve the bioavailability of OA. While water solubility of OA was increased by the above-mentioned preparation method, there was little change in the permeability of $O A$ itself, which may account for its low bioavailability.

Cubic liquid crystal nanoparticles (LCNP) are now being investigated as a universal carrier for drug delivery ${ }^{[15-19]}$ to improve the oral bioavailability of the drug because of high encapsulation efficiency, drug loading and bio-affinity ${ }^{[20]}$. In our previous study, we prepared curcumin and piperine-loaded LCNP using a precursor injection method. The results showed that the oral bioavailability of LCNP-curcumin was significantly improved ${ }^{[21]}$. Based on that work, in this study we explored the possibility of LCNP as a carrier system for a topical formulation of OA. In addition, in this this study we also evaluated the in vitro permeability of the drug through rat skin and determined the anti-inflammatory activity of the LCNP gel. 


\section{Materials And Methods}

\section{Material}

Phytantriol (purity $>98 \%$ ) was purchased from TCl (Tokyo, Japan). OA was acquired from Acetar Bio-tech Inc (Shanxi, China). Lutrol F127, propylene glycol, glycerin, carbopol 934 (CP), HPMC K4M, ethanol, were all acquired from Shanhe Pharmaceutical Accessories Co., Ltd. (Anhui, China). Sodium dodecyl sulfate was purchased from Hunan Er-kang Pharmaceutical Co., Ltd. (Hunan, China). Azone was purchased from Tianmen scientific Pharmaceutical Co., Ltd. (Hubei, China). Triethanolamine was given as a gift from Jiangxi a-Hitech scientific Pharmaceutical Co., Ltd. (Jiangxi, China). Ultrapure water was produced by using a Milli-Q purification system from Millipore (Massachusetts, USA). Acetonitrile and methanol (both HPLC grade) were purchased from Merck (New Jersey, Germany). Standards of OA (CAS: 508-02-1, > 95\%) were purchased from Nation Institutes for Food and Drug Control (Beijing, China). All materials were used as received.

\section{Methods}

\section{Preparation of OA loaded LCNP}

The nanoparticles were prepared using the precursor method described in our previous study ${ }^{[21]}$. Drugs and excipients were weighed according to the prescriptions in Table 1. The phytantriol and OA were dissolved in $2.5 \mathrm{~mL}$ of absolute ethanol simultaneously with sonication at $80 \%$ of maximum power for 30 minutes. The organic phase was added dropwise to the aqueous phase $(20 \mathrm{~mL})$ containing stabilizer F127 under agitation at $500 \mathrm{rpm}$ for 3 hours to form a crude dispersion, which was then sonicated (Misonix XL2000, Misonix Inc., Farmingdale, N.Y.) in a pulsed mode (0.5 s pulse interrupted by $0.5 \mathrm{~s}$ interrupt) at $40 \%$ of maximum power for 10 minutes to produce a milky dispersion and to reduce particle size. In addition, LCNP containing OA with loading amounts of $6 \%, 8 \%$ and $10 \%$, respectively, were prepared and their effects on encapsulation efficiency were investigated. The OA-loaded LCNP dispersion obtained was freeze-dried to be a dry and free-flowing powder.

Table 1

Different Formulations for OA-loaded LCNP

\begin{tabular}{|lllll|}
\hline S.No. & Formulations parameters & \multicolumn{3}{l|}{ Formulations } \\
\cline { 2 - 5 } & & OAF1 & OAF2 & OAF3 \\
\hline 1 & Phytantriol $(\mathrm{mg})$ & 70 & 75 & 80 \\
\hline 2 & F127(mg) & 30 & 25 & 20 \\
\hline 3 & OA $(\mathrm{mg})$ & 6 & 8 & 10 \\
\hline
\end{tabular}

Particle size, micrographs and crystal cell parameter measurements of OA-loaded LCNP 
The size of LCNP was measured using dynamic light scattering (Nano patica SZ100, H field, Iwate Prefecture, Japan); we used an average of five measurements. Micrograghs of the nanoparticles were

investigated by scanning electron microscopy (SEM; 1430 VP, Leo, Sauerlach, Germany) ${ }^{[21]}$. In determining the size of the nanoparticles, the cubic phase nanoparticle suspension was first placed on silicon water through a capillary tube, then allowed to dry at room temperature. Thereafter, they were coated with platinum for 2 minutes in the ion sputtering instrument. The coated samples were measured at an acceleration voltage of $15 \mathrm{kV}$. Average particle size and polydispersity index were measured by photon correlation spectroscopy at a $90^{\circ}$ angle at $25^{\circ} \mathrm{C}$.

Polarizing light microscopy (BX41P, Olympus, Tokyo, Japan) with a (MATS-U55S, Tokaihit, Shizuoka, Japan) heater set between a range of $20-60^{\circ} \mathrm{C}^{[21]}$ was used in this study The crystal cell parameter measurement used a Bruker Nanostar small-angle X-ray scattering (SAXS) camera with pinhole collimation for point focus geometry. The sample was placed in the quartz capillary tube, and the optics and sample chamber were placed under vacuum to minimize air scatter. The results were normalized for sample transmission after the background was subtracted and integrated using Bruker AXS software. The diffraction patterns obtained were converted to plots of intensity versus q value.

\section{Zeta potential analysis of OA-loaded LCNP}

The nanoparticle surface charge was measured by determining the zeta potential using a Zetasizer (3000HS Malvern Instruments, UK). The LCNP suspension was diluted with double distilled water (1:100, $\mathrm{w} / \mathrm{w}$ ) to obtain a uniform dispersion before testing, and the conductivity was then measured at $25^{\circ} \mathrm{C}{ }^{[22]}$. All values calculated were the average of three independent measurements (mean \pm standard deviation).

\section{Drug entrapment efficiency (DEE) and drug loading (DL) of OA-loaded LCNP}

Nanoparticles were embedded in a Sephadex G-50-filled microcolumn under a centrifugal force of 500 rpm for 5 minutes and then eluted with the same volume of ultrapure water. The eluate was obtained by centrifugation at 2,000 rpm for 5 minutes. After repeating the same procedure for five times, the eluate was then collected and dissolved in methanol. The drug content of the eluate was measured in triplicate by a validated HPLC method. DEE of LCNP was calculated as follows: DEE $(\%, w / w)=$ Total amount of drug- Amount of drug in supernatant $\times 100 /$ Total amount of drug. DL $(\%, w / w)=$ Initial drug - free drug/Mixed lipid $\times 100$

\section{In vitro release from OA-loaded LCNP}

The in vitro release was measured by a dialysis method. Briefly, the nanoparticles and the suspensions were placed in the dialysis bag (Biosharp, Hefei, China) with a molecular cut off of $14 \mathrm{kDa}$, and the dialysis bag was suspended in $20 \mathrm{~mL}$ of release medium (containing $0.5 \%$ sodium lauryl sulfate) at $37^{\circ} \mathrm{C}$ while the speed of the magnetic agitation was set at $300 \mathrm{rpm} .100 \mu \mathrm{L}$ of sample was withdrawn at predetermined time interval and replaced with fresh medium. The sample content was then determined 
by the in vitro HPLC method. The HPLC apparatus used for the quantitation consisted of an Agilent 1200 HPLC system (Agilent, USA) with DAD detector. Compounds were separated on a Kromasil RP-C18 column (4.6 mm $\times 250 \mathrm{~mm}$, Akzo Nobel, Stockholm, Sweden) using a flow rate of $1.0 \mathrm{~mL} / \mathrm{min}$ with a fixed mobile phase [acetonitrile and $0.1 \mathrm{moL} \cdot \mathrm{L}^{-1}$ ammonium acetate (55:45)]. The wavelength was set at $210 \mathrm{~nm}$. The injection volume was $10 \mu \mathrm{L}$ and the retention time of OA was determined to be $27.665 \mathrm{~min}$.

The LCNP matrix contained the same amount of ingredients but without OA, and this was used as a blank for comparison. Calibration curves plotted for OA showed good linearity, with a correlation coefficient $\left(r^{2}\right)$ of 0.9993 in the concentration ranges tested $\left(10.42 \sim 104.2 \mu \mathrm{g} \cdot \mathrm{mL}^{-1}\right)$.

This analytical method has been validated in accordance with the guidelines of the International Conference on Harmonization of Technical Requirements for Registration of Pharmaceuticals for Human Use $(\mathrm{ICH})$. Parameters such as accuracy, reproducibility, and stability were verified separately. The relative standard deviations (RSD) of intra and inter day analyses were $<3 \%$, and the accuracy of the method was verified with the average recovery rates of $99.02 \%$.

\section{Preparation of OA-LCNP-based gel}

After optimizing the OA-LCNP method, the topical gel loaded with OA-LCNP was prepared by a dispersion swelling technique using Carbopol 934 and HPMC K4M. All excipients were weighed as shown in Table 2, and then Carbopol 934 and HPMC K4M were simultaneously dispersed in water and allowed to stand for 4 hours to properly swell the polymer. OA-LCNP powder (equivalent to $0.5 \mathrm{mg} / \mathrm{g}$ ) was then added to the polymer gel under constant one-way agitation to avoid air bubbles. Triethanolamine was then added to the gel mixture for crosslinking between Carbopol 934 polymer and HPMC K4M to form a gel. Then, propylene glycol and glycerin were added to the gel to balance its viscosity, azone was added to increase the permeability of the gel, and finally the $\mathrm{pH}$ was adjusted to a skin $\mathrm{pH}$ of $7.4 \pm 0.1$ by addition of $0.1 \mathrm{~N}$ $\mathrm{NaOH}^{[23]}$. In addition, a conventional OA-loaded gel (NG) was prepared in the same manner without OAloaded LCNP.

Table 2

Formulation table for OA-LCNP-based Gel

\begin{tabular}{|llllll|}
\hline S.No. & Ingredients & $\begin{array}{l}\text { OA- } \\
\text { LCNPG1(w/w) }\end{array}$ & $\begin{array}{l}\text { OA- } \\
\text { LCNPG2(w/w) }\end{array}$ & $\begin{array}{l}\text { OA- } \\
\text { LCNPG3(w/w) }\end{array}$ & NG(w/w) \\
\hline 1 & CP 934 & $1.5 \%$ & $1.0 \%$ & $0.5 \%$ & $1.0 \%$ \\
\hline 2 & HPMC K4M & $0.5 \%$ & $1.0 \%$ & $1.5 \%$ & $1.0 \%$ \\
\hline 3 & glycerol & $10 \%$ & $10 \%$ & $10 \%$ & $10 \%$ \\
\hline 5 & propylene glycol & $5 \%$ & $5 \%$ & $5 \%$ & $5 \%$ \\
\hline 7 & Triethanaolamine & $2 \%$ & $2 \%$ & $2 \%$ & $2 \%$ \\
\hline
\end{tabular}

\section{Physical appearance and formulation $\mathrm{pH}$}


Basic physicochemical parameters were screened for different formulations. The color, transparency, homogeneity and appearance of the prepared gel were visually inspected. The $\mathrm{pH}$ of the $1 \%$ aqueous solution of the gel was measured by a pH meter (Mettler Toledo, Switzerland). One gram per meter of gel was dissolved in $100 \mathrm{~mL}$ of distilled water and stored for $2 \mathrm{~h}^{18}$. The $\mathrm{pH}$ of each formulation was measured in triplicate and the average was calculated.

\section{Viscosity and Spreadability analysis of OA-LCNP-loaded topical gel}

In the rheological properties test, all developed formulations were placed in a beaker, placed in the rotor, and then rotated at $10 \mathrm{rpm}\left(25-27^{\circ} \mathrm{C}\right)$ in a Brookfield viscometer (V-550, Thermo). To measure the spreadability of the formulated OA-LCNP gel, we introduced 1 gram of gel per gram between two slides, held the pre-weighed plate over the gel and gradually added more weight until the gel stopped spreading. The final cumulative weight and total time required for gel diffusion were measured and recorded separately. The spreadability of the gel was then calculated according to the formula, i.e., the total weight applied and the gel mass were compared by the time ${ }^{[23]}$. Spreadibility = Mass $\times$ Length $/$ Time

\section{Ex vivo drug permeation studies of OA-LCNP-loaded topical gel}

The measurements of drug permeation were performed using a Franz diffusion cell to evaluate the $O A$ release profile from each formulation. The surface area of the release membrane was $1.971 \mathrm{~cm}^{2}$, and the receiving chamber volume was $18.54 \mathrm{~mL}$. Before performing the permeability study, the abdominal skin of the rat was obtained and processed. The subcutaneous tissue was carefully removed by surgery, and the dermal side was wiped with isopropyl alcohol to remove the attached fat layer. The skin was cleaned again with distilled water and then stored at $-18^{\circ} \mathrm{C}$. During the permeability test, the skin was placed between the donor and recipient compartments of the Franz cells with the stratum corneum side facing the donor compartment and the dermis side facing the receptor compartment ${ }^{[24]}$.

One gram (equivalent to $0.5 \mathrm{mg} \mathrm{OA}$ ) of gel (NG, OA-LCNP G1, OA-LCNP G2, OA-LCNP G3, and NG) was added in to the donor compartment. The receptor compartment was filled with PBS (pH 7.4) (containing $0.5 \%$ sodium lauryl sulfate) and kept in full contact with the dermis layer, the temperature was controlled at $37^{\circ} \mathrm{C}$, and the speed of magnetic stirring was performed at $400 \mathrm{rpm} .2 \mathrm{~mL}$ of the receptor solution was withdrawn at an interval of $0.5,1,2,3,6,9,12 \mathrm{~h}$. After each sampling, an equal volume of PBS was simultaneously added to the receptor compartment to maintain volume. Each sample was filtered through a $0.45 \mu \mathrm{m}$ polyamide membrane filter (Satorius, Germany), and then the OA content was determined by HPLC. The concentration in the sample taken and the percentage of drug release of the preparations were calculated.

\section{Analysis of permeability OA-LCNP-loaded topical gel}


For each formulation, the percentage of drug permeated through the skin $\left(\mu \mathrm{g} \cdot \mathrm{cm}^{-2}\right)$ was plotted as a function of time. By dividing the slope of the linear portion of the graph by the diffusion cell area $\left(\mu \mathrm{g} \cdot \mathrm{cm}^{-}\right.$ ${ }^{2} \cdot \mathrm{h}^{-1}$ ), the drug flux (permeability) at steady state (Jss) was calculated. The permeability coefficient (Kp) was calculated by dividing Jss by the initial concentration of the drug in the donor cell $\left(\mathrm{cm} \cdot \mathrm{h}^{-1}\right)$. The enhancement rate $(\mathrm{Er})$ was calculated by dividing the Jss of each formulation by the Jss of the control formulation ${ }^{[24]}$.

\section{Drug release kinetics studies OA-LCNP-loaded topical gel}

The data obtained from in vitro drug release studies were plotted as percent drug release versus time (Zero-order equation), logarithm of drug residue versus time (First-order equation), percentage of drug release versus square root of time (Higuchi model equation ), and the logarithmic relationship between logarithmic drug release and logarithmic time (Korsmeyer equation) to assess drug release mechanisms. The standard values for the release mechanism are listed in Table 5.

\section{In vivo anti-inflammatory studies of OA-LCNP-loaded topical gel}

Anti-inflammatory studies were performed in adult male Wistar rats weighing $150-250 \mathrm{~g}$ according to a previously reported technique ${ }^{[25]}$. Briefly, rats were randomly divided into three groups, 1 experimental group and two control groups ( $n=6$ per group). Group I received topical saline application (control group), Group II received commercially available mometasone furoate gel (standard group), and Group III received LCNP-based gel formulation (test group). The animal study was approved by the Institutional Animal Ethics Committee with registration number SYXK 2017-0044 (Jiangsu Province, China).

The left hind paw of the rat was first marked directly above the tibia-tar junction. The paw was immersed in the electrolyte column every time until the marker was fixed in order to measure the volume of the constant paw. The test formulation was applied to the left hind paw of the rat for 30 minutes before carrageenan-induced inflammation. The initial paw volume (Vo) of the rats was determined just prior to injection of carrageenan, and the volume increase due to fluid excretion was noted from the digital display, and then $0.1 \mathrm{~mL}$ of $1 \%$ carrageenan solution was injected into the saline which located at the subplantar area of the left hind paw of the rat. The paw volume $(\mathrm{Vt})$ was then assessed after $1,2,4,8,12$ and 24 hours, respectively. The edema rate and inhibition rate of each group are calculated as follows:

Edema Rate $(E \%)=(\mathrm{Vt}-\mathrm{Vo}) / \mathrm{Vo} \times 100$

Inhibition Rate $(1 \%)=(E c-E t) / E c \times 100$

Where Ec is the edema rate of control group, and Et is the edema rate of the treated group.

\section{Results And Discussion}

\section{Screening of excipients}


When developing the LCNP system, suitable liquid, lipid and surfactants were selected for use, and were generally considered safe (GRAS).

\section{Surfactant screening}

In order to prepare stable LCNPs, two surfactants were screened, Lutrol F127 and glyceryl monooleate. We found that LCNP was more soluble in Lutrol F127 compared to glyceryl monooleate, so it was the surfactant chosen for OA-loaded LCNP.

\section{Partitioning behavior of LCNP in various lipids}

We showed that LCNP had a higher partition in Lutrol F127 (logP = 3.265) compared to glyceryl monooleate $(\log P=1.892)$. Therefore, Lutrol F127 was chosen to formulate a LCNP system loaded with OA.

\section{Screening of formula technology}

We compared 2 methods to prepare LCNP; the precursor injection method and the solvent emulsion evaporation technique. The precursor-injection method exhibited the smallest particle size (273 \pm 18.92 $\mathrm{nm})$ and highest entrapment efficiency (70.12 $\pm 2.12 \% \mathrm{w} / \mathrm{w})$ compared to the solvent emulsification evaporation technique, which resulted in a particle size of $543 \pm 32.93 \mathrm{~nm}$ and entrapment efficiency of $45.18 \pm 2.08 \% \mathrm{w} / \mathrm{w}$.

\section{Optimization of process parameters}

Several process parameters were determined prior to preparation of the LCNP. The ratio of phytantriol to F127, surfactant concentration, agitation time, agitation speed and homogenization cycle were optimized over a wide range to enable selection of the optimum formulation. The ratio of phytantriol to F127 was optimized with the measurement of particle size and encapsulation efficiency. The ratios of phytantriol to F127 were assumed to be 70:30, 80:20 and 90:10, respectively. The results showed that the ratio of phytantriol to $\mathrm{F} 127$ was $80: 20$ with the smallest particle size $(138 \pm 10.21 \mathrm{~nm})$ and the optimal encapsulation efficiency of the nanoparticles is $72.39 \pm 2.36 \% \mathrm{w} / \mathrm{w}$, as shown in Fig. 1 . The quality and efficacy of cubosomes were significantly affected by the properties and concentrations of the surfactant. Surfactants were preferentially distributed in the interfacial region because of the surface active properties. Therefore, the concentration of F127 was optimized with 1, 1.5, 2, and $2.5 \%$ w/v, respectively. The surfactant was gradually added during emulsification, and the concentration of the F127 was optimized by measuring drug retention and particle size after emulsification for $24 \mathrm{~h}$. A clear transparent dispersion was obtained at a F127 concentration of $1.5 \% \mathrm{w} / \mathrm{v}$ with a minimum particle size of $145 \pm$ $11.82 \mathrm{~nm}$ and a retention efficiency of $71.83 \pm 2.43 \% \mathrm{w} / \mathrm{w}$. The results of surfactant concentrations of $2.0 \%$ and $2.5 \% \mathrm{w} / \mathrm{v}$ both revealed the aggregation of translucent and opaque particles. Therefore, the surfactant concentration of $1.5 \% \mathrm{w} / \mathrm{v}$ was determined ${ }^{[21]}$.

The effects of different stirring speeds between 500, 1000, 1500, rpm were screened, and 1000 rpm was chosen because it had the lowest particle size $142 \pm 11.83 \mathrm{~nm}$ and the best encapsulation efficiency 
$71.93 \pm 2.93 \%$ w/w as shown in Fig. 2 .

In addition, stirring time between 15,30,45, and 60 min was also screened and it was determined that 30 min was optimal to obtain the lowest particle size. The homogenization cycle was screened based on the measurements of particle size and encapsulation efficiency for 3, 6 and 9 cycles. The results showed that as the number of cycles increased, the particle size first decreased and then increased slightly. This may be due to the fact that as the homogenization cycle increased, the system temperature gradually decreased, resulting in an increase in kinetic energy, thus the particles reassemble during preparation ${ }^{[23]}$. Six cycles achieved a minimum particle size of $142 \pm 9.98 \mathrm{~nm}$ and an optimal drug retention of $72.17 \pm$ $2.83 \%$ w/w as shown in Fig. 3.

When assessing evaluated parameters, we developed different formulations to take into account optimization of parameters, i.e. 0, 1 (optimization parameters), and the +1 level was certain as given in Table 1. The LCNP loaded with OA was successfully prepared by the precursor injection method, and the parameters such as particle size, encapsulation efficiency, zeta potential and drug release in vitro were characterized. The LCNP loaded with OA showed particle size in the range of $129 \pm 12.11 \mathrm{~nm}$ to $272 \pm$ $21.83 \mathrm{~nm}$. The minimum particle size was $129 \pm 12.11 \mathrm{~nm}$ of the formulation OAF1, which is the certain condition for local delivery of LCNP as shown in Table 3.

Table 3

Particle size, Zeta potential, and polydispersity index of OA-LCNP

\begin{tabular}{|lllllll|}
\hline Formulations & $\begin{array}{l}\text { Drug } \\
\text { loaded(\%, } \\
\mathbf{w} / \mathrm{w})\end{array}$ & $\begin{array}{l}\text { Particle } \\
\text { size } \\
(\mathrm{nm}) \pm\end{array}$ & $\begin{array}{l}\text { Zeta } \\
\text { potential }\end{array}$ & $\begin{array}{l}\text { Polydispersity } \\
\text { index }\end{array}$ & $\begin{array}{l}\mathrm{DEE}(\% \\
\mathbf{W} / \mathbf{w}) \pm \text { SD }\end{array}$ & $\begin{array}{l}\mathrm{DL}(\% \\
\mathbf{w} / \mathrm{w}) \pm \text { SD }\end{array}$ \\
\hline OAF1 & 6 & $129 \pm$ & $-18.3 \mathrm{mV}$ & 0.322 & $73.18 \pm$ & $12.31 \pm$ \\
& & 12.11 & & & 3.21 & 0.41 \\
\hline OAF2 & 8 & $159 \pm$ & $-21.2 \mathrm{mV}$ & 0.218 & $70.93 \pm$ & $14.12 \pm$ \\
& & 17.87 & & & 3.28 & 0.32 \\
\hline OAF3 & 10 & $272 \pm$ & $-19.9 \mathrm{mV}$ & 0.436 & $68.31 \pm$ & $13.11 \pm$ \\
\hline
\end{tabular}

The particle size of OAF1 and OAF2 decreased as the concentration of the surfactant increased, but we noted an eventual increase. This may be due to an increase in the homogenization cycle and OA content. As the homogenization cycle increased, the kinetic energy of the system increased, causing particle aggregation ${ }^{[23]}$. The smaller size may help improve penetration of the drug through the biofilm and aid in targeting. These are considered to be important criteria for topical drug delivery systems that use loaded nanoparticles. In general, the particle size of the nanoparticles should be as small as possible to penetrate the skin more easily, especially in the case of LCNP. Under some circumstances, entrapment efficiency can be considered to be less important than particle size for LCNP formulation development. 
The values of zeta potential were in the range of -18.3 to $-21.2 \mathrm{mV}$ for all three formulations. A high zeta potential is related to the storage stability of the nanoparticles, which may indicate that the nanodispersions may not aggregate. We found that the zeta potential increased and then decreased (OAF1 < OAF2).

Determination of the particle size and the distribution of nanoparticles require measurement of the polydispersity index (PDI). A sample having a polydispersity value of less than 0.7 indicates that the nanoparticles are substantially uniformly dispersed. The results showed that the values of the PDI of the three formulations ranged from 0.218 to 0.436 , with the formula CPF2 showing a minimum PDI of 0.218 . The results showed that the PDI value decreased with an increase of phytantriol and then increased. The shape and surface morphology of the LCNP were observed by scanning electron microscope (SEM). The average particle size of the cubic nanoparticles was $152 \mathrm{~nm}$ as determined by dynamic light scattering (DLS) (Fig. 4).The SEM image revealed that the particles were cube-shaped with Pn3m space group and stacked in 3D (Fig. 5).

The polarity of LCNP was also studied using a polarizing microscope, and the dispersion of LCNP was observed using polarizing microscopy without treatment. The polarity image of the cubosome nanoparticles was shown using dark field due to the optical isotropic characteristic of LCNP, generally indicative of the formation of the cubic phase. However, heating at $55^{\circ} \mathrm{C}$, the anisotropic image appeared with the presence of fan-like texture, indicative of the formation of the Hk phase rather than the cubic phase, which was due to the transformation of the crystal structure (Fig. 6) ${ }^{[27]}$. In addition, when the temperature decreased to $20^{\circ} \mathrm{C}$, the dark field of the image reappeared, indicative of the formation of the cubic phase.

SAXS was used to determine the internal structure of the cubosome nanoparticles. Figure 7 shows a plot of intensity versus the scattering vector, $\mathrm{q}$, obtained from formulations prepared using phytantriol and F127. The OA-loaded LCNP displayed four peaks, where the first two were more intense than the second pair. The relative position of the peaks was in accordance with the bicontinuous cubic phase structure with Pn3m space group, indicative of the cubosome nanoparticles with a D-type cubic nanostructure with lattice parameters of $67.4 \AA$.

The encapsulation efficiency of the LCNP loaded with OA was in the range of $68.31 \% \pm 2.86-73.18 \% \pm$ $3.21 \mathrm{w} / \mathrm{w}$. Encapsulation efficiency is related to the crystallinity of the lipid nanoparticles; the more phytantriol in the formulation mixture, the higher the entrapment efficiency. The drug loading of OAloaded LCNP was in the range of $12.31 \% \pm 0.41$ to $14.12 \% \pm 0.32 \mathrm{w} / \mathrm{w}$. Compared with OAF2 $(84.56 \% \pm$ $0.19)$ and OAF3 $(81.22 \% \pm 0.15)$, OA loading LCNP (OAF1) showed the largest in vitro drug release rate of $85.49 \% \pm 0.21$ within 12 hours as shown in Fig. 8. The in vitro release of OA from the LCNP dispersion was biphasic with an initial burst effect followed by a gradual release of OA. The initial release may be due to the presence of un-embedded drug in the LCNP dispersion, or it may be due to the presence of dissolved forms of the drug in the outer shell of the liquid lipid and lead to burst in the initial phase. 
However, release may occur due to corrosion or diffusion of the substrate ${ }^{[27]}$. The drug provided by the initial burst release immediately produces a rapid therapeutic effect, and the permeability of the drug was improved, while the sustained release of the drug is prolonged and the concentration of the therapeutic agent at the site of action is maintained. This clearly shows that sustained release can be obtained by using this formulation with a single application.

\section{Characterization of OA-LCNP-loaded topical gel}

The preparations of OA-LCNP-loaded topical gel were lucid, clear, and uniformed in texture, and the $\mathrm{pH}$ ranges of 7.1-7.4 which could easily be tolerated on skin without any stimulation. On account of lowest concentration of HPMC compared with OA-LCNP-G2 (64,000-71,000 cps) and OA-LCNP-G3 (55,000$63,000 \mathrm{cps})$, OA-LCNP-G1 had the lowest viscosity $(72,000-79,000 \mathrm{cps})$. OA-LCNP-G1 can be spread easily for application compared to OA-LCNP-G2 since its slightly lower viscosity. Results for LCNP-loaded gel characterization are provided in Table 4. Values for percentage in vitro drug release up to $12 \mathrm{~h}$ for OALCNP-G1, G2, and G3 are $84.93 \%, 86.78 \%$, and $87.89 \%$, respectively, as given in Fig. 9 .

Table 4

Characterization parameters for OA-LCNP-loaded gel

\begin{tabular}{|llllll|}
\hline S.No. & Formulation & pH & Viscosity(cps) & $\begin{array}{l}\text { Spreadability } \\
\left(\mathbf{g} \cdot \mathbf{c m} \cdot \mathbf{m i n}^{-1}\right)\end{array}$ & $\begin{array}{l}\text { Drug content } \\
(\% \mathrm{w} / \mathrm{w})\end{array}$ \\
\hline 1. & OA-LCNP-G1 & 7.3 & $72,000 \sim 79,000$ & $258.98 / 2.35$ & $96.12 \pm 0.31$ \\
\hline 2. & OA-LCNP-G2 & 7.4 & $64,000 \sim 71,000$ & $241.33 / 2.43$ & $97.32 \pm 0.29$ \\
\hline 3. & OA-LCNP-G3 & 7.3 & $55,000 \sim 63,000$ & $247.91 / 2.41$ & $96.89 \pm 0.12$ \\
\hline 4. & NG & 7.4 & $67,000 \sim 73,000$ & $246.38 / 3.13$ & $97.46 \pm 0.41$ \\
\hline
\end{tabular}

The permeation study suggested that permeability parameters such as steady-state flux (Jss), permeability coefficient (Kp), and increased ratio were significantly higher in both of the OA-LCNP-G1 and OA-LCNP-G2 formulations, compared to OA-LCNP-G3. The cumulative amount of the permeated drug at the end of $12 \mathrm{~h}$ was $371.5,384.7$, and $429.8 \mu \mathrm{g} / \mathrm{cm}^{2}$ with a steady state flux (Jss) of 188.5, 195.2, and $218.0 \mu \mathrm{g} / \mathrm{cm}^{2} / \mathrm{h}$ for OA-LCNP-G1, OA-LCNP-G2, and OA-LCNP-G3, respectively, as shown in Fig. 10. Value of permeability coefficient is high for OA-LCNP-G1 $(1.16 \mathrm{~cm} / \mathrm{h})>$ OA-LCNP-G3 $0.96 \mathrm{~cm} / \mathrm{h})>$ OA-LCNP-G2 $(0.98 \mathrm{~cm} / \mathrm{h})$. There was an increased ratio of OA-LCNP-G3 and OA-LCNP-G2, 1.16 and 1.36, respectively, compared to the OA-LCNP-G1 gel preparation.

The in vitro drug release from the optimized OA-LCNP-G1 was best explained by Higuchi's equation, as the plots showed the highest linearity $\left(R^{2}=0.989\right)$, followed by first order $\left(R^{2}=0.924\right)$ and zero order $\left(R^{2}=\right.$ 0.887). The corresponding plot of (log \% cumulative drug release vs log time) for the Korsmeyer-Peppas equation indicated good linearity $\left(R^{2}=0.963\right)$. The value $r^{2}$ of Peppas equation indicated good linearity 
showing irregular (non-Fickian) diffusion which means that drug release was controlled by more than one process, and the diffusion may be controlled as well as swelling controlled release as shown in Table 5.

Table 5

Release kinetic parameters for OA-loaded LCNP gel (OA-LCNP-G2)

\begin{tabular}{|llllllll|}
\hline \multicolumn{2}{|l}{ Zero order } & \multicolumn{2}{l}{ First order equation } & \multicolumn{2}{l|}{ Peppas equation } & \multicolumn{2}{l|}{ Higuchi equation } \\
\hline$k$ & $R^{2}$ & $k$ & $R^{2}$ & $k$ & $R^{2}$ & $k$ & $R^{2}$ \\
\hline 3.124 & 0.887 & 0.0416 & 0.924 & 0.6048 & 0.963 & 18.212 & 0.989 \\
\hline
\end{tabular}

\section{In vivo anti-inflammatory studies}

The anti-inflammatory activity of the optimized preparation for OA-LCNP-loaded topical gel was evaluated using the carrageenan-induced hind paw inflammation method on Wistar rats. The percentage inhibition value of OA-LCNP gel (test) was also compared to marketed mometasone furoate gel (standard) as displayed in Table 6. OA-LCNP gel decreased the inflammation to a certain degree and also revealed a sustained effect for a prolonged period.

Table 6

In vivo anti-inflammatory activity: carrageenan-induced hind paw edema

\begin{tabular}{|lllllllll|}
\hline Treatment & $\begin{array}{l}\text { Anti- } \\
\text { inflammatory } \\
\text { activity }\end{array}$ & $\mathbf{0}$ & $\mathbf{1}$ & $\mathbf{2}$ & $\mathbf{4}$ & $\mathbf{8}$ & $\mathbf{1 2}$ & $\mathbf{2 4}$ \\
\cline { 2 - 9 } & Time & & & & & & \\
\hline Control & Edema rate & 0.00 & 34.12 & 43.87 & 44.32 & 45.87 & 46.98 & 40.92 \\
& & \pm 0.02 & \pm 0.02 & \pm 0.02 & \pm 0.01 & \pm 0.02 & \pm 0.02 & \pm 0.02 \\
\hline Test & Edema rate & 0.00 & 32.28 & 39.56 & 36.81 & 36.13 & 35.41 & 25.28 \\
& & \pm 0.01 & \pm 0.02 & \pm 0.02 & \pm 0.02 & \pm 0.02 & \pm 0.02 & \pm 0.02 \\
& Inhibition & & 5.61 & 7.81 & 17.32 & 20.83 & 24.89 & 45.26 \\
& rate(\%) & & & & & & & \\
Standard & Edema rate & 0.00 & 31.72 & 34.11 & 31.87 & 26.13 & 21.09 & 17.42 \\
& & \pm 0.01 & \pm 0.02 & \pm 0.02 & \pm 0.02 & \pm 0.02 & \pm 0.02 & \pm 0.02 \\
\hline
\end{tabular}

\section{Conclusions}

In this study, OA-loaded LCNP was prepared by the precursor method, resulting in a well-characterized OALCNP gel preparation. The gel was shown to be effective in a rodent carrageenan-induced hind paw inflammation with sustained efficacy after a single application.

\section{Declarations}

Ethics approval and consent to participate 
All treatments were carried out according to Guangdong Institute of Traditional Chinese Medicine, Ethical Committee Acts.

Consent for publication

Not applicable.

Availability of data and materials

All data generated or analysed during this study are included in this published article.

Competing interests

The authors declare that they hava no competing interests.

Funding

This project was supported by the Natural Science Foundation from education department of Jiangsu Province, China (Grant Number: 19KJB360001) and Changzhou Science and Technology and Information Bureau (Grant Number: CJ20189003 and CJ20190011).

Authors' contributions

Z.Q.S. and S.G.P. conceptualized this study, and should be considered co-first authors. Z.Q.S. and S.G.P ran the experiments. Z.Q.S., L.L.W.and S.L. analyzed the data and contributed to the writing of the paper. All authors read and approved the final manuscript.

Acknowledgements

The authors would like to express their gratitude to EditSprings (https://www.editsprings.com/) for the expert linguistic services provided.

Authors' information

${ }^{1}$ Changzhou Institute of Industry and Technology, Changzhou 213164, China. ${ }^{2}$ Changzhou Institute of Technology, Changzhou 213022, China. ${ }^{3}$ Key Laboratory for Soft Chemistry and Functional Materials of Ministry Education, Nanjing University of Science and Technology, Nanjing, China. ${ }^{4}$ Guangdong Provincial Institute of Traditional Chinese Medicine, Guangzhou 510095, China. ${ }^{5}$ Wuxi Hospital of Traditional Chinese Medicine, Wuxi 214071, China.

\section{References}

1. Liu J. Pharmacology of oleanolic acid and ursolic acid. J Ethnopharmacol. 1995;49:57-68. 
2. Dzubak P, Hajduch M, Vydra D, et al. Pharmacological activities of natural triterpenoids and their therapeutic implications. Nat Prod Rep. 2006;23:394-411.

3. Pollier J, Goossens A. Oleanolic acid. Phytochem. 2012;77:10-5.

4. Somova LI, Shode FO, Ramnanan P, et al. Antihypertensive, antiatherosclerotic and antioxidant activity of triterpenoids isolated from Olea europaea, subspecies africana leaves. J Ethnopharmacol. 2003;84:299-305.

5. Singh GB, Singh S, Bani S, et al. Anti-inflammatory activity of oleanolic acid in rats and mice. J Pharm Pharmacol. 1992;44:456-8.

6. Kurek A, Nadkowska P, Pliszka S, et al. Modulation of antibiotic resistance in bacterial pathogens by oleanolic acid and ursolic acid. Phymed. 2012;19:515-9.

7. Lee W, Yang EJ, Ku SK, et al. Anti-inflammatory Effects of Oleanolic Acid on LPS-Induced Inflammation In Vitro and In Vivo. Inflam. 2013;36(1):94-101.

8. Bednarczyk-Cwynar B, Wachowiak N, Szulc M, et al. Strong and Long-Lasting Antinociceptive and Anti-inflammatory Conjugate of Naturally Occurring Oleanolic Acid and Aspirin. Front Pharmacol. 2016;7:1-18.

9. Liu J. Oleanolic acid and ursolic acid: research perspectives. J Ethnopharmacol. 2005;100:92-4.

10. Jeong DW, Kim YH, Kim HH, et al. Dose-linear pharmacokinetics of oleanolic acid after intravenous and oral administration in rats. Biopharm Drug Dispos. 2007;28:51-7.

11. Gao D, Tang S, Tong Q. Oleanolic acid liposomes with polyethylene glycol modification: promising antitumor drug delivery. Int J Nanomedicne. 2012;7:3517-26.

12. Tong HH, Du Z, Wang GN, Chan HM, et al. Spray freeze drying with polyvinylpyrrolidone and sodium caprate for improved dissolution and oral bioavailability of oleanolic acid, a BCS Class IV compound. Int J Pharm. 2011;404:148-58.

13. Chen Y, Liu J, Yang X, et al. Oleanolic acid nanosuspensions: preparation, in-vitro characterization and enhanced hepatoprotective effect. J Pharm Pharmacol. 2005;57:259-64.

14. Jiang QK, Yang XX, Du P, et al. Dual strategies to improve oral bioavailability of oleanolic acid: Enhancing water-solubility, permeability and inhibiting cytochrome P450 isozymes. Eur J Pharm Biopharm. 2016;99:65-72.

15. Esposito E, Cortesi R, Drechsler M, et al. Cubosome dispersions as delivery systems for percutaneous administration of indomethacin. Pharm Res. 2005;22(12):2163-73.

16. Gan L, Han S, Shen J, et al. Self-assembled liquid crystalline nanoparticles as a novel ophthalmic delivery system for dexamethasone: Improving preocular retention and ocular bioavailability. Int $\mathrm{J}$ Pharm. 2010;396:179-87.

17. Rizwan SB, Assmus D, Boehnke A, et al. Preparation of phytantriol cubosomes by solvent precursor dilution for the delivery of protein vaccines. Eur J Pharm Biopharm. 2011;79:15-22.

18. Yang Z, Tan Y, Chen M, et al. Development of amphotericin B-loaded cubosomes through the SolEmuls technology for enhancing the oral bioavailability. AAPS Pharm Sci Tech. 2012;13(4):1483- 
91.

19. Mei LL, Xie XC, Jing H, et al. A novel design for stable self-assembly cubosome precursormicroparticles enhancing dissolution of insoluble drugs. Drug Dev Ind Pharm. 2017;43:1239-43.

20. Deshpande S, Singh N. Influence of Cubosome Surface Architecture on its Cellular Uptake Mechanism. Langmuir. 2017;33:3509-16.

21. Tu YS, Fu JW, Sun DM, et al. Preparation, characterisation and evaluation of curcumin with piperineloaded cubosome nanoparticles. J Microencapsul. 2014;31:307-16.

22. Sanad RA, Abdelmalak NS, Elbayoomy TS, et al. Formulation of a novel oxybenzone-loaded nanostructured lipid carrier (NLCs). AAPS Pharm Sci Tech. 2010;11(4):1684-94.

23. Nagaich U, Gulati N. Nanostructured lipid carriers (NLC) based controlled release topical gel of clobetasol propionate: design and in vivo characterization. Drug Deliv Transl Res. 2016;6:289-98.

24. Thatipamula RP, Palem CR, Gannu R, et al. Formulation and in vitro characterization of domperidone loaded solid lipid nanoparticles and nanostructured lipid carriers. Daru. 2011;19(1):23-32.

25. Hagit A, Shlomo L, Tomer I, et al. Induction, treatment and prevention of eczema vaccinatum in atopic dermatitis mouse models. Vaccine. 2017. http://dx.doi.org/10.1016/j.vaccine.

26. Landh T. Phase behavior in the system pine needle oil monoglyceridespoloxamer 407-water at 20.degree. J Phys Chem. 1994;98:8453-67.

27. Ata S, Rasool A, Islam A, et al. Loading of Cefixime to $\mathrm{pH}$ sensitive chitosan based hydrogel and investigation of controlled release kinetics. Int J Bio Macromol. 2019;12:1-29.

\section{Figures}

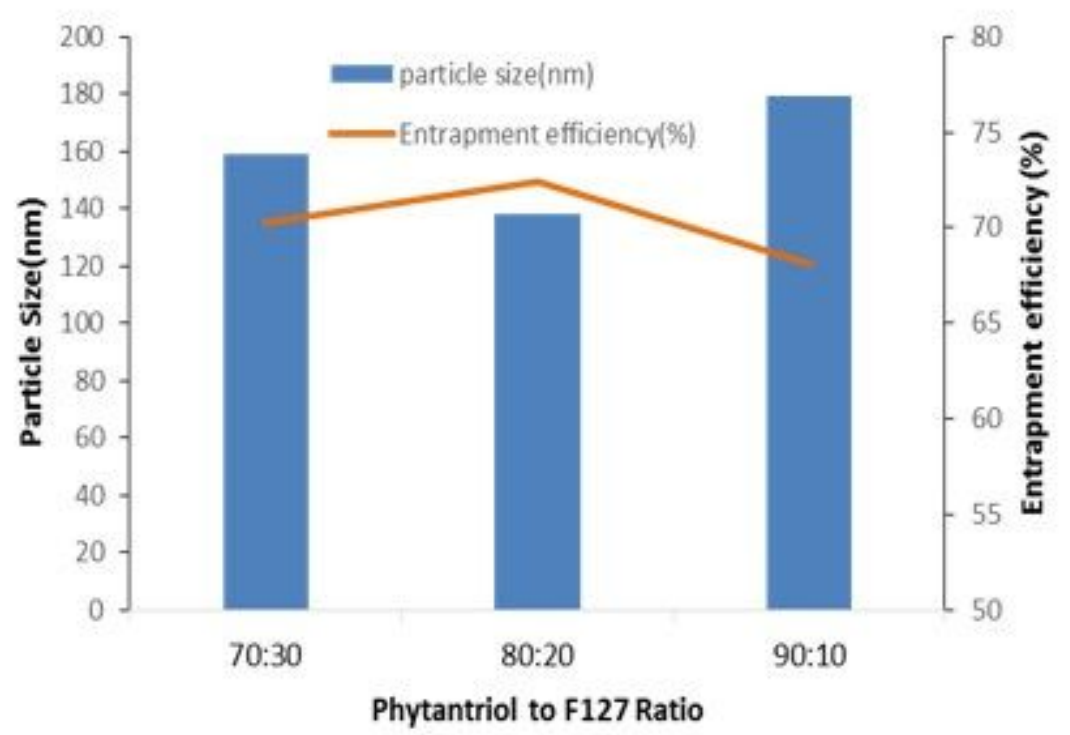

\section{Figure 1}

Phytantriol to F127 ratio on the basis of particle size and entrapment efficiency 


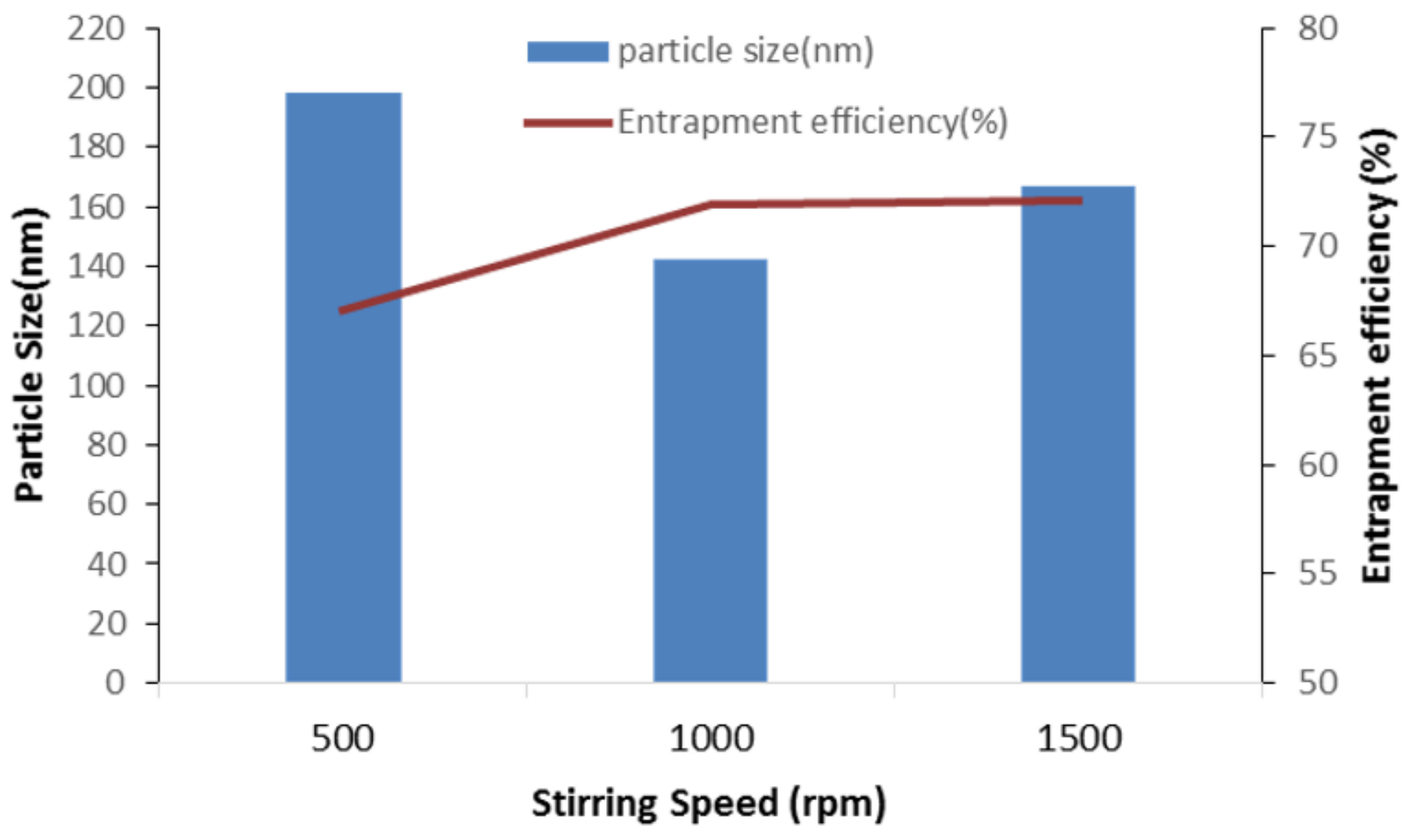

Figure 2

Optimization of stirring speed on the basis of particle size and entrapment efficiency

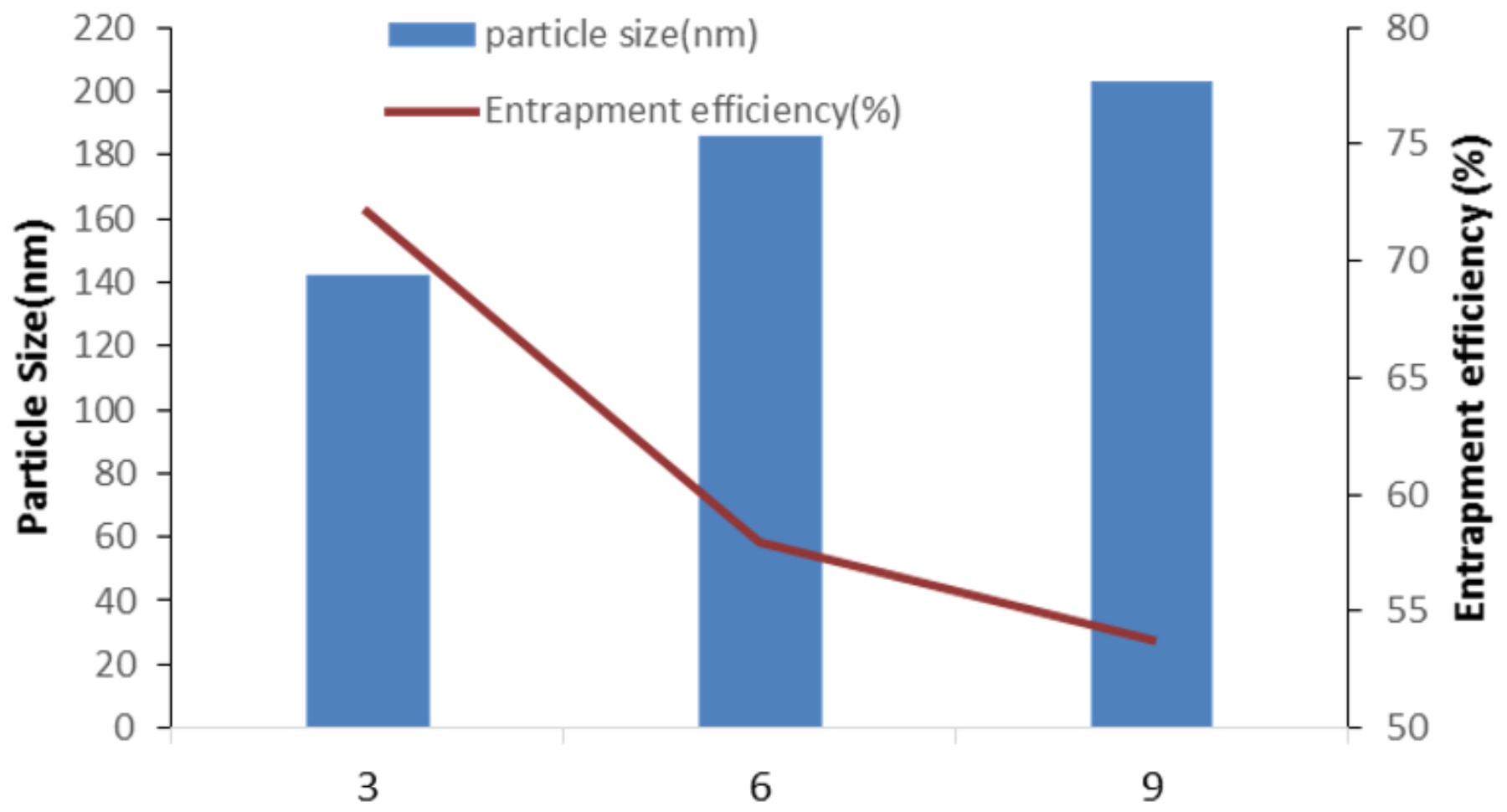

Homogenization cycles 
Figure 3

Optimization of homogenization cycles on the basis of particle size and entrapment efficiency

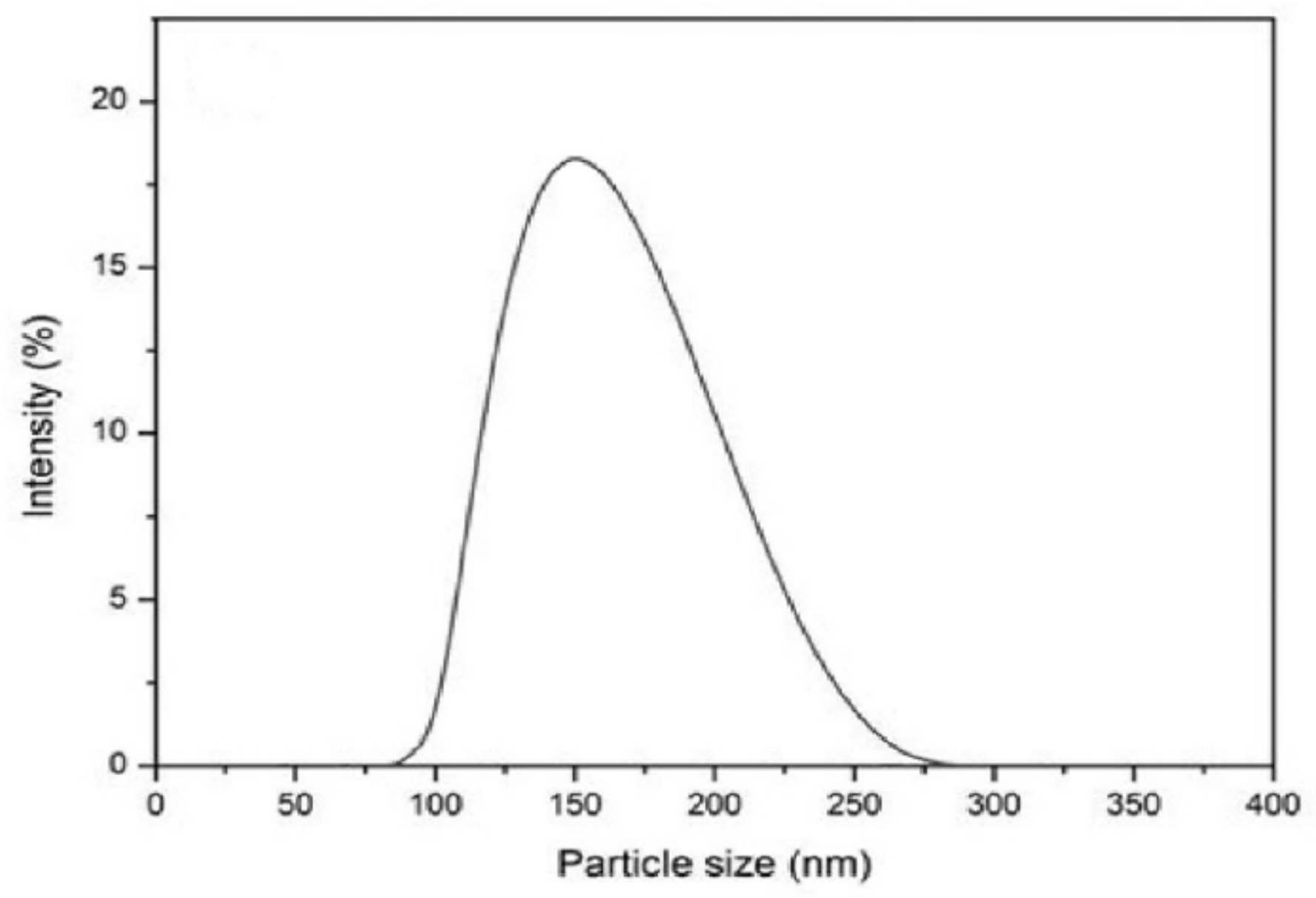

Figure 4

Particle size distribution of OA-loaded LCNP 


\section{A1394 SEI 15.0KV $\times 65$, ODO 100}

\section{Figure 5}

Morphology of OA-loaded LCNP by scanning electron microscope (Magnification 65,000 )

(a)

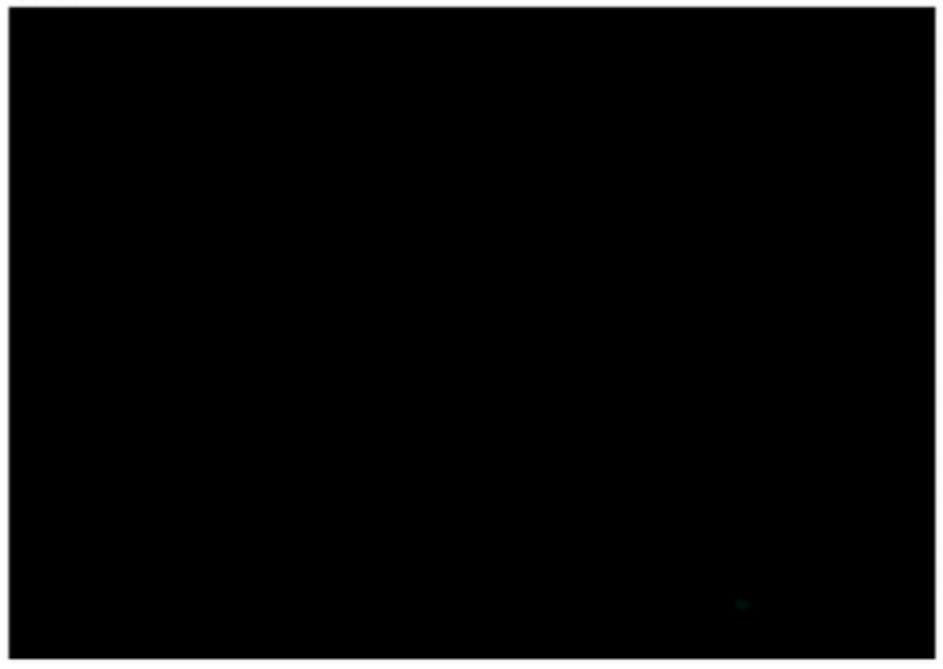

(b)

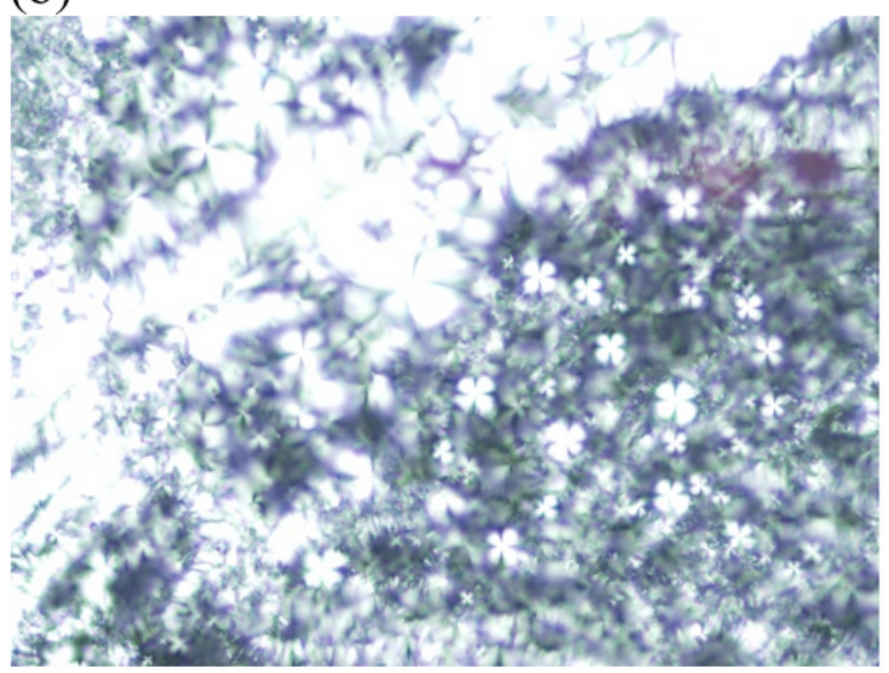

Figure 6

(a) Polarizing light micrograph of OA-loaded LCNP at $20^{\circ} \mathrm{C}$ and (b) the cubosome at $55^{\circ} \mathrm{C}$. 


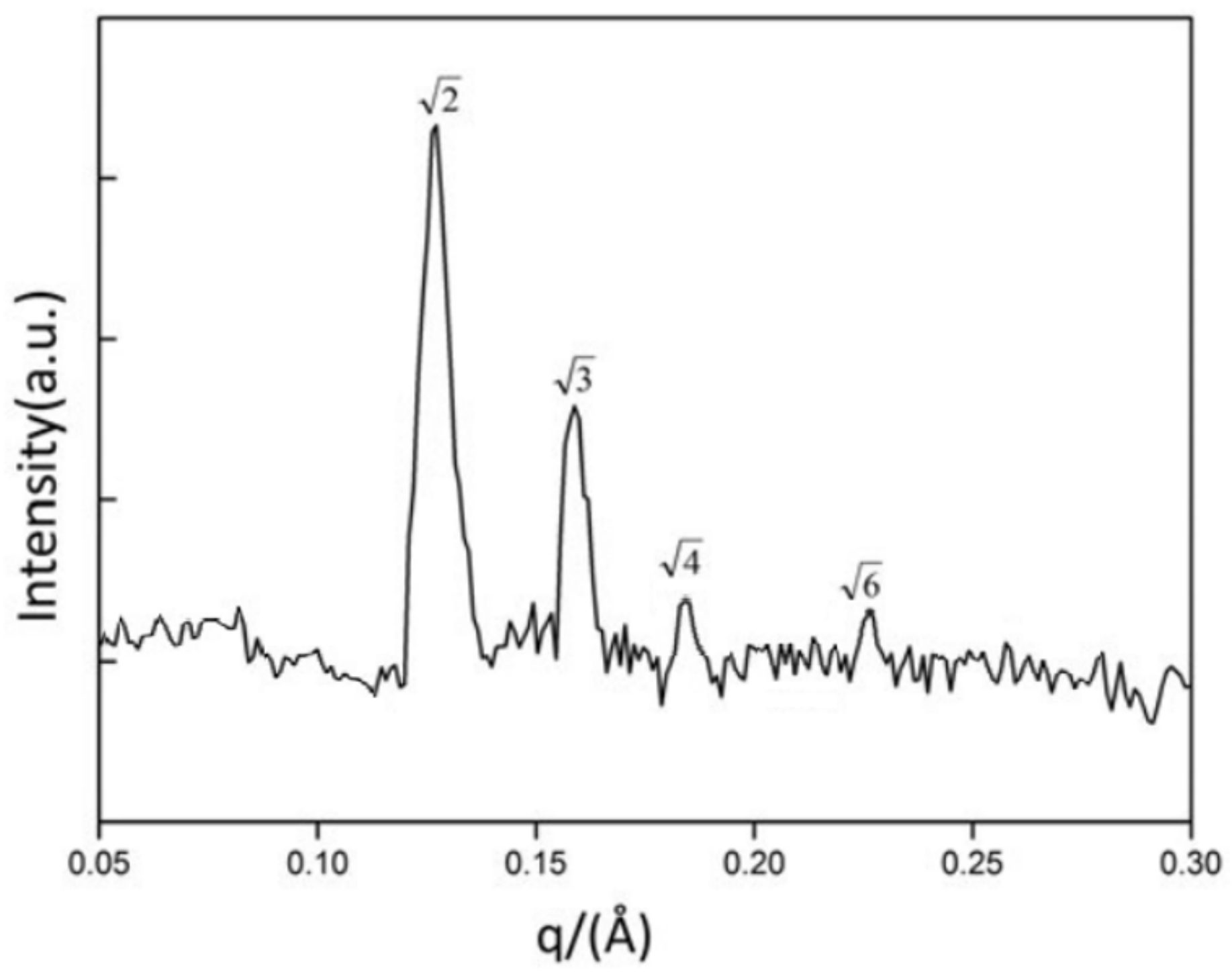

Figure 7

Intensity versus q plot from SAXS measurement of OA-loaded LCNP. 


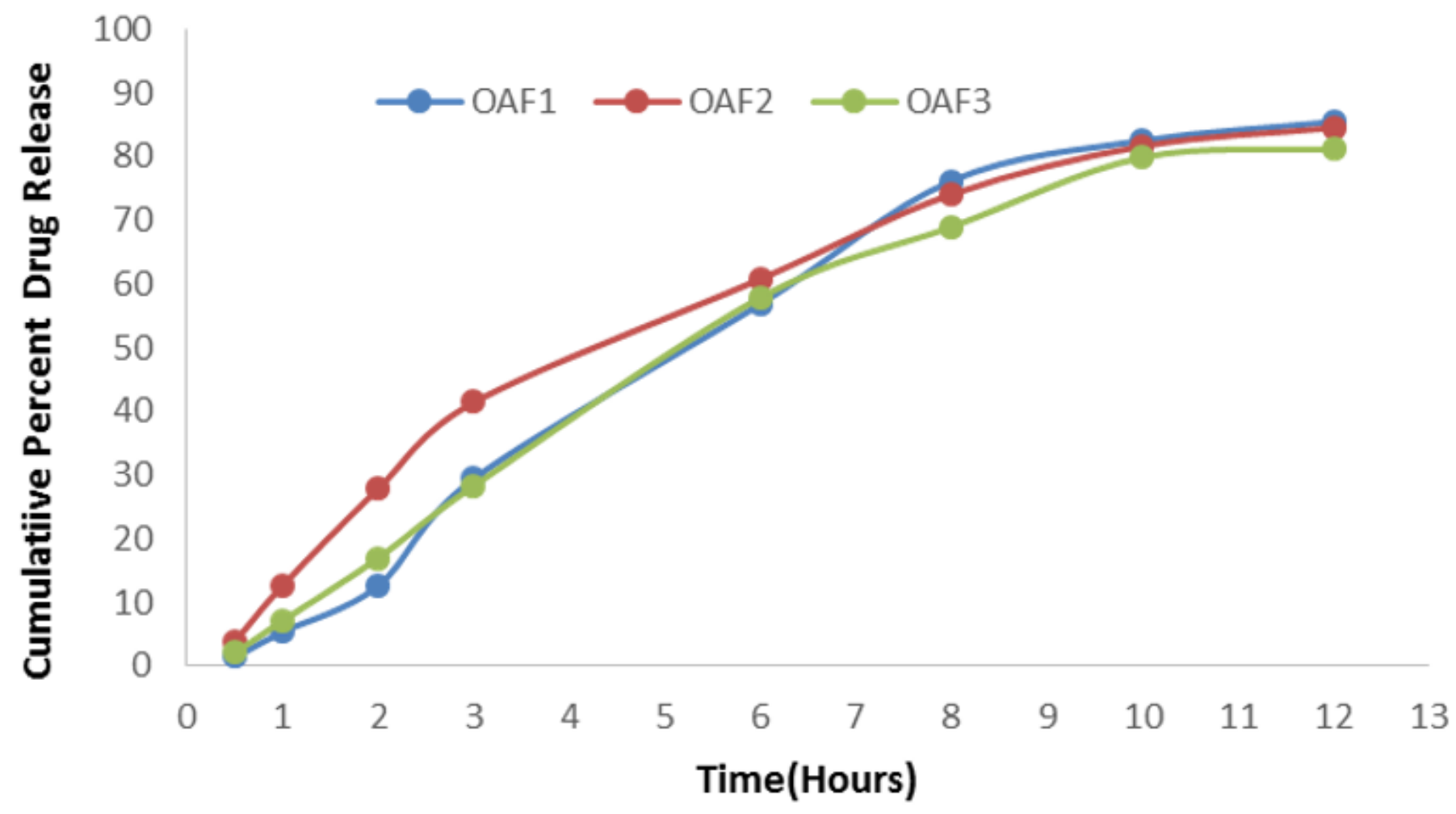

Figure 8

In vitro drug release profiles of OA-loaded LCNP up to $12 \mathrm{~h}$

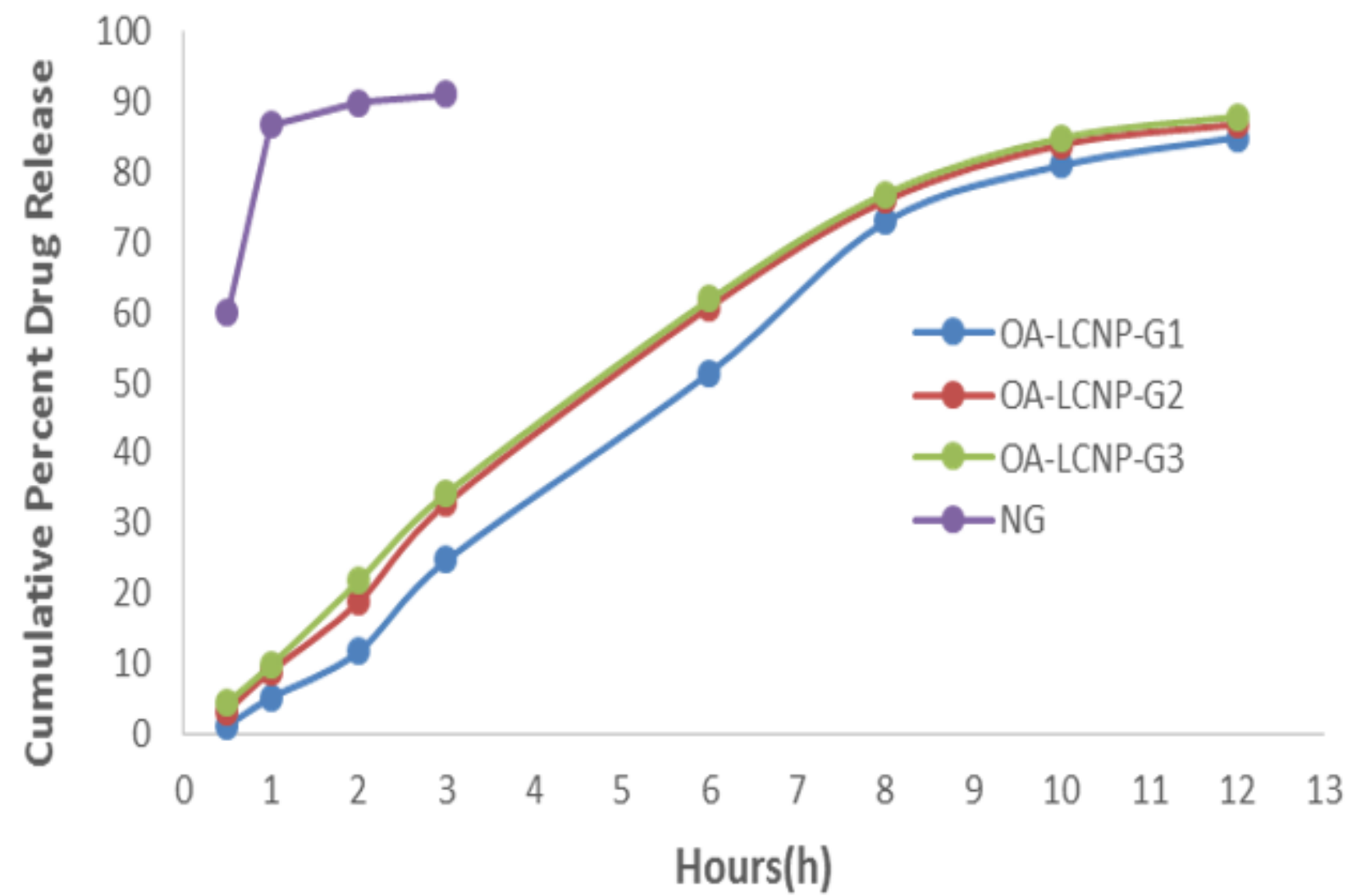


Figure 9

In vitro drug release profiles of OA-LCNP-loaded topical gels up to $12 \mathrm{~h}$

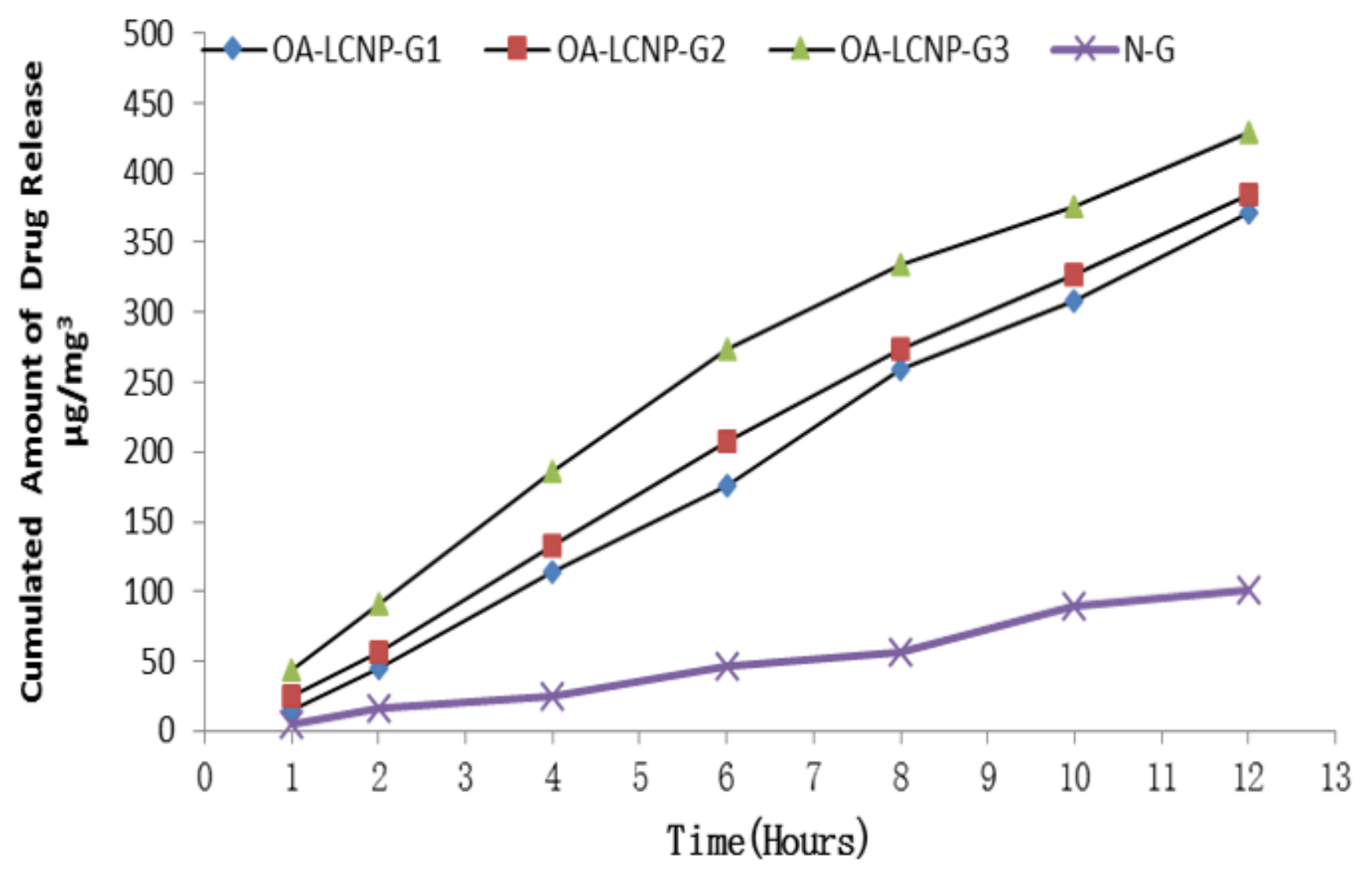

Figure 10

Ex vivo permeation results of different OA-LCNP-loaded gel formulations 